months, probably owing to the vitamin agitation raised by the press and to the vitaminisation of the margarine.

"The effect of parenteral administration of carotin oil proves to be slower than that of injection of liver oil preparations. A series of extensive investigations of the power of distinction is accounted for, the test subjects being 28 prisoners of the State prison in Nyborg. The power of distinction is tested regularly throughout a period of nine months. One group of prisoners evidently presented seasonal oscillations in the power of distinction, which failed to appear in a control group of prisoners who received the same diet with a supplement of milk."

\title{
CORRESPONDENCE
}

\section{TUBERCULIN IN TREATMENT OF TRACHOMATOUS KERATITIS}

\section{To the Editors of THE BRITISH JOURnal OF OPHTHALMOLOGy.}

SIRS,--May I ask if you can grant the space for the following brief account on the subject of the title:-My object is to draw attention to a new field in which tuberculin proved to play an important part, namely, the treatment of trachomatous manifestations in the cornea. These are, briefly, infiltrations (superficial and deep), pannus and ulcers. The fact that severe trachoma of the conjunctiva sometimes occurs without apparent change in the cornea has often struck me. On the other hand, one often sees cases of severe trachomatous keratitis of the recurrent type with only mild trachomatous conjunctivitis. The usual treatment of such cases up till the present has been to treat energetically the condition of the conjunctiva and apply atropine ointment, which in many cases ended in failure. This led me to the belief that trachomatous keratitis was only a manifestation of a specific disease. Having successfully treated and cured many obstinate cases with tuberculin alone (without any local applications), I came to the conclusion that the specific disease was latent tuberculosis, the foci of which remained quiescent and inactive, at least temporarily, but could be activated by exposure to unfavourable conditions, e.g., illness, extensive re-infection and exhaustion. The presence of these latent foci is demonstrable with any of the tuberculin tests as mentioned in most text books on bacteriology. Having obtained a positive reaction one proceeds to give tuberculin subcutaneously twice weekly at first and once weekly later, starting always with minute 
doses; the fundamental rule is never to provoke a febrile reaction of any importance. Personally I begin with $1 / 2000 \mathrm{mg}$. and increase gradually to $1 / 10 \mathrm{mg}$. which I continue giving once a week for a long time (over a year). Lately $I$ have been injecting tuberculin in the lids deep in the submuscular layer with very favourable results. Here I begin with $1 / 10,000 \mathrm{mg}$. and add $1 / 10,000 \mathrm{mg}$. to each subsequent dose. This method gives much quicker effect and enables one to observe the reaction objectively, the amount of swelling of the lid being an estimate of the toxicity. of the given dose. The proper dose should only produce a mild fleeting swelling. To guard against mixed infection during this treatment, I give the patient 2 per cent. mercurochrome drops to instil at home.

Results of Treatment.-Lacrymation stops, photophobia disappears and vision improves appreciably before the second injection. Objective signs take longer to disappear, and the rate of progress differs in the different cases. A case of mine had severe pannus with total infiltration of the left cornea and vision, when I fist saw her (October 10), was C.F. at $50 \mathrm{~cm}$. On October 17, under tuberculin treatment alone, vision improved to C.F. at $1 \mathrm{~m}$., on the 20th L.V. $=4 / 60$, on the 30 th $6 / 60$ and on the first day of November 6/36. Another case had fleshy pannus in the upper part of the cornea with punctate ulcers which had been recurring for three months. I saw her first on April 27, the ulcers healed after the first injection and the thick fleshy pannus disappeared entirely to the naked eye on July 10 .

The condition of the lid improved along with that of the cornea, so that there was no need to apply any medicine locally, far less to interfere surgically. Indeed I have found that local interference would retard the progress.

Among other conditions I have successfully treated with tuberculin were multiple styes, chronic blepharitis, phlyctenular keratoconjunctivitis and especially deep infiltrations of the cornea of obscure nature. Two of my cases had their left corneae entirely opaque as a result of curious deep infiltrations which resisted all sorts of treatment for three months. On tuberculin treatment alone, the opacity in each got gradually lighter and finally disappeared. This took one month in one case and 39 days in the other. There were no signs of iridocyclitis.

Your obedient servant,

$$
\begin{aligned}
& \text { M. A. L. HAsSOUNA, D.O.M.S., } \\
& \text { Tutor of Govt. O.H. Rodel Farag. }
\end{aligned}
$$

PONT DE KUBBA,

CAIRO.

Deceinber 5, 1937. 